\title{
48. The Sterility of Barley Caused by Some Environmental and Internal Conditions
}

\author{
By Isamu STow \\ Meguro Laboratory of the Brewing Science Research Institute \\ (Comm. by Tetsu Sakamura. M. J. A., March 13, 1972)
}

The heavy sterility of the barley happens frequently in Japan, and several investigators have studied the cause of it. The writer has been engaged in this problem for years, and he stated previously1) that it might occur by the combination of the anthesis in sheath and the high humidity in floret, both caused by the influence of weather conditions.

1. Weather conditions and sterility. To determine the relation between the sterility and weather conditions, it is necessary to find out the day of anthesis when the sterility might have occurred. Therefore, the fertile grains neighbouring to the sterile one were examined for their size and shape to distinguish the age of the young grains.*) Thus the date on which the sterility might have occurred

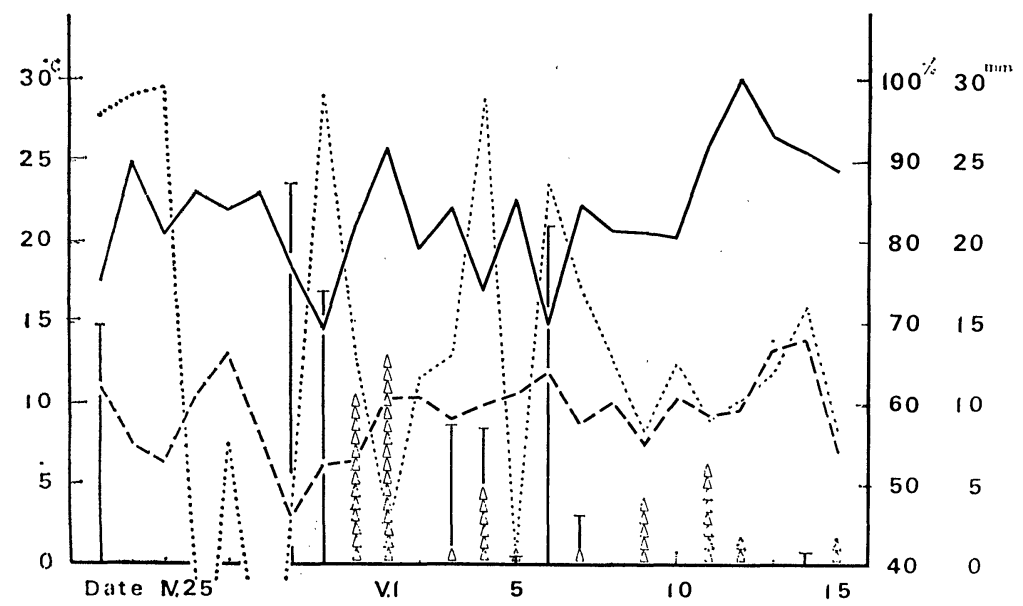

Fig. 1. The number of spikes with sterile grains was plotted on the date on which anthesis took place, showing the relation between the sterility and the weather conditions. New Golden; the experimental farm of Nirasaki Hop Preparation Plant of Kirin Brewery, Nirasaki, Yamanashi Prefecture; 1971.

-: Maximum temperature, ----: Minimum temperature, …...: Humidity, $T$ : Precipitation, $\triangle$ : Spike with sterile grain.

*) It is not so difficult for those experienced in pollination tests to estimate roughly the age of the young grain from 3 to 15 days after fertilization. 
was presumed, because it should be the same day when anthesis and fertilization took place.

Then the dates of anthesis, i.e. the dates on which sterility occurred, were plotted on the weather chart, and it was found that the plots are concentrated on the humid days preceded by several days of warm weather (Fig. 1) ; this indicates that the sterility is related more or less to the weather conditions.

2. Emergence and sterility. The sterility is always accompanied by inferiority of the emergence of spike, and the less the emergence the higher the sterility. To ascertain this relationship, following measurements were carried out.

Here, "the degree of emergence" is defined as "the distance from the top of the sheath to the lower end of the spike" which is obtained by the difference between the length of sheath and the length of topinternode of the culm.

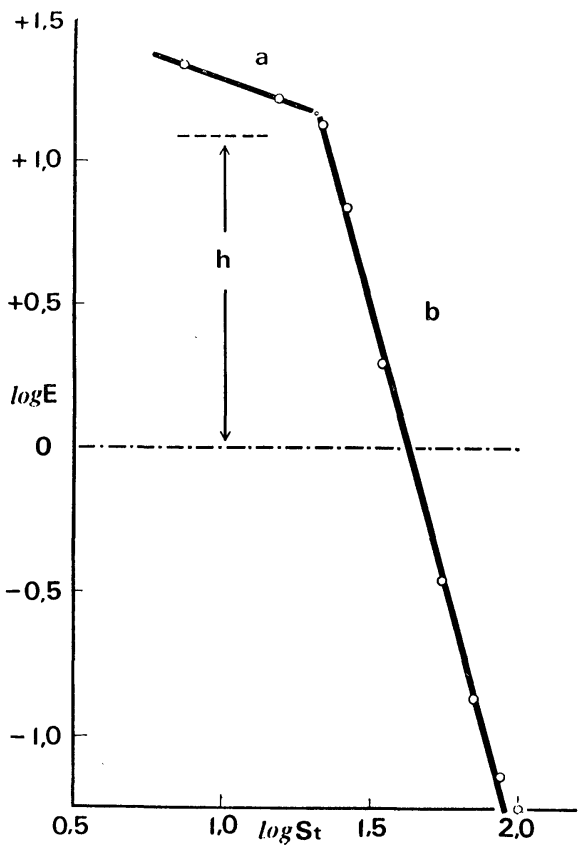

Fig. 2. Relation between emergence and sterility. The logarithm of degree of emergence (log E) and the logarithm of percentage sterility of spikes ( $\log \mathrm{St}$ ) are plotted on a rectangular co-ordinate. The data are based on 154 spikes with sterile grains collected from 8 places of different environmental conditions in Yamanashi and Tochigi Prefectures. h: Probable growing length of the topinternode after anthesis. 
The logarithm of the degree of emergence was plotted on the ordinate and the logarithm of percentage sterility on the abscissa of a rectangular co-ordinate. When the sheath length was shorter than the length of top-internode, the plot was placed on the positive (upper) side of the origin, while in the reverse case it was placed on the negative (under) side. The figure thus obtained (Fig. 2) consists of two straight lines, which suggests the existence of an intimate connection between the sterility and the emergence of spike. It is noteworthy that the break-point falls on $\mathbf{h}$ which is the probable growing length of top-internode after anthesis. So the line a, the upper part, indicates the anthesis after the perfect emergence, while $b$, the lower part, implies the anthesis in imperfect- or non-emergence.

Thus it is obvious that the inferior emergence may be at least one of the most important factors involved in the sterility.

3. The cause of inferior emergence. From the results mentioned above, we can suggest that the sterility might have occurred through the anthesis in sheath. So, it is very important to find out the mechanism to cause the elongation hindrance of the top-internode which induces the inferior emergence. To investigate such abnormal growth of the top-internode, the normal growing function of organs were studied.

As the growth phenomenon follows an exponential function, we shall obtain a straight line when the logarithm of growth length against the corresponding time is plotted on a rectangular co-ordinate. In the case of barley plant in respect of any vegetative organ tested, however, it never gives a single straight line, but consists of four straight lines connected at three points (Fig. 3). The three points for different organs occur simultaneously, so that it seems that the growth of every organ is controlled uniformly by a single source. On close examinations, it was found that the three connecting points correspond to the time of the meiosis (sporogenesis, S-point), of the generative cell differentiation (gametogenesis, G-point) and of the fertilization (zygogenesis, Z-point), respectively. Needless to say, they are very important periods of growth phenomena in the sexual physiology. From these facts, we can suppose that the sexual organ must be playing a leading or at least an important role in the growth control of all organs. That is to say, the growth of sexual organ may be an autonomous function while that of the vegetative ones is a heteronomous, at least after the sexual differentiation took place. Perhaps such control may be performed by the production of some hormone- or retardant-like substance from the sexual organ or tissue, according to its respective developing stages. This supposition may be supported more or less by the following preliminary test. 


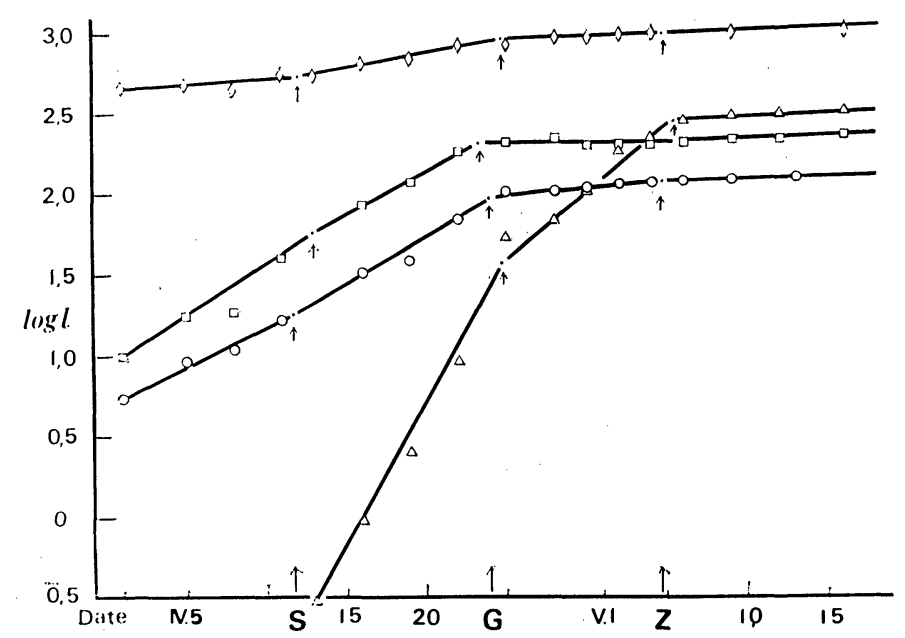

Fig. 3. Growth curves of different organs in barley, Hanna Kargin; observed at Nagano Experimental Station of the Brewing Science Research Institute, Nagano Pref., 1949. It is shown that all curves consist of four lines each, the breakpoints occurring simultaneously. $\log \mathrm{L}$ : Logarithm of length, S: Period of meiosis (sporogenesis), G: Period of generative cell differentiation (gametogenesis), Z: Period of anthesis (fertilization, zygogenesis).

$\diamond$ : Grass height, $\square$ : Sheath (flag leaf), $\bigcirc$ : Spike, $\triangle$ : Top-internode, $\uparrow$ : Connection point (break-point).

The lower part of a young culm with the spike at the meiotic stage was kept at $15 \sim 16^{\circ} \mathrm{C}$ for days by covering it with a coil of fine vinyl tube through which water was circulated. The size of the coil was $2 \mathrm{~cm}$ in inside diameter and 3 or $5 \mathrm{~cm}$ in height, which was just enough to cover the spike in sheath; the upper and lower ends of the coil were sealed loosely with vinyl tape. The other culms of the same plant were kept free from such equipment as control. The whole plants in the pots were placed in a little glass house at $2.9 \sim 25.5^{\circ} \mathrm{C}$, daily average being $8.9 \sim 17.2^{\circ} \mathrm{C}$, from March 25,1971 . After several days, control culms showed somewhat better growth than the treated ones, and the difference became distinct as the days went by. None of the treated culms reached perfect emergence, while most of controls showed normal emergence, although both gave fertile grains with few sterile ones, presumably because of the lack of high humidity in this case.

From these results, we can suppose that the temperature affected the sexual organ, and this in turn affected to the vegetative organs.

Summary. The sterility of barley frequently encountered here in Japan has been studied.

1) Sterility is closely related to weather conditions: it occurs 
when anthesis takes place on a humid day preceded by several days of warm weather.

2) Sterility is correlated with inferiority of emergence at the time of anthesis.

3) Growth curves of vegetative organs consist of four lines connected at three points, which correspond to the periods of sporogenesis, gametogenesis and zygogenesis-the very important periods of growth phenomena in the sexual physiology. It thus indicates that the sexual organ should play an important role to control the growth of all organs.

4) Experimental attemperation $\left(15 \sim 16^{\circ} \mathrm{C}\right)$ of the spike at and after the meiotic stage resulted in inferior emergence.

Acknowledgement. The writer wishes to his sincere thanks to Mr. Y. Kato of Otawara and Mr. M. Katsura of Sano, Tochigi Prefecture, for their kind guidance and assistance to collect the samples. He also expresses his gratitude to Kirin Brewery Co. Ltd. for permission to collect the valuable samples in their field and for generous provision of the meteorological records.

\section{Reference}

1) Stow, I.: Plant Physiology, 5, 94 (1966) (in Japanese). 